\title{
Effect of the Gravity on the Sagging Breasts of Women
}

Giovanni Alcocer, Priscilla Alcocer \& Carlos Márquez

Independent Researches, Guayaquil, Ecuador.Email: giov_alc_science@hotmail.com

Crossref

DOI: 10.38177/ajast.2020.4319

\section{ABSTRACT}

The level of the breast deformation is not related to the fact that the breasts are large or small. For large breasts, it depends if the breasts are very soft (small E, high $\Delta \mathrm{y}$ ) or firm (high E, small $\Delta \mathrm{y}$ ) (Young's modulus $\mathrm{E}$, vertical displacement $\Delta \mathrm{y}$ ) in order that a significant deformation occurs. For small breasts, there is generally no significant deformation. The deformation of the breast increases for very soft breasts and decrements for very firm breasts. Therefore, a good bra of breast can minimize this effect. This paper is related to the respective analysis of the relation of the variables mentioned before by using the equation $\Delta y=\frac{\rho g y^{2}}{E}$.

Besides, biomechanical models developed using the Finite Element Method (FEM) and programming can be used to model the relationship between different types of tissues (breasts) and with the application of forces or movements to know possible deformations $(1 \ldots .9,11,12,13,15,16)$. It can help predict mechanical deformations of the tissue during medical operations and derive and quantify the properties of the breasts with the observed deformations (15). The theory of the finite elasticity (10) is used to implement the finite element model. We need to know the biomechanical properties, boundary conditions and a configuration of a normal breast and deformed. It can be very useful when it is done a diagnostic examination (mammography, magnetic resonance) and the patient moves from one position to another and the breasts are compressed. Thus, masses of difficult observation could be visible between those images. It may be possible to have a biomechanical model of the breast. Then, it can be used for breas cancer diagnosis and for studies of the gravity effects on light and heavy breasts and to study the effects of compression on radiographic tests Furthermore, it is possible to use silicone gel phantom (1), (3), for breast experimentation. So, the phantom (breast) could be subjected to differen weights and analyze the elasticity of the breast to support different forces. It can be done together with the respective simulations. Therefore, we can compare the experimental results (vertical displacements) with the simulations. Then, one could validate the biomechanical model and the simulation program. In addition to the phantom and simulations, we could make a graph of $\sigma$ (strain) versus $\mathrm{C}$ ( $\Delta \mathrm{y}$ displacement rate) . Finally, we can get the limit points of yield. As we said before and with these researches, we can design fasterners (17) or other material with the aim that the breast does not reach the limit point of yield.

Keywords: Effect of gravity, Breast deformation, Soft breast, Firm breast, Young's modulus E, Vertical displacement $\Delta y$, Biomechanical model Fasterners (bra), Silicone phantom, Graph $\sigma$ versus $€$.

\section{Introduction}

The fall of the breasts is an inevitable and natural process that happens to all women at some point in her life, except for those with very small breasts. The breasts do not have muscles, and thus they cannot be strengthened. They have fat, ligament and connective tissue. When the breasts are attracted down by the gravitational force $\left(\mathrm{F}_{\mathrm{g}}\right)$, ligaments and skin are stretched and the breasts fall. However, the fact that no sagging breasts remain depends on the elasticity of the skin and ligaments (Cooper's ligament), which are determined by the genes, the diet and the aging process.

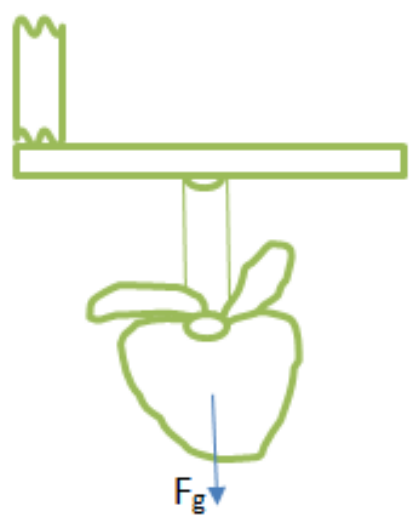

Fig.1 Apple (which is analogous to the breast) suspended of its stem (which is analogous to the Cooper's ligament) 
Apparently, one would think that the large breasts are easier to fall because the gravity pulls them harder. But the fact that they fall depends on both the weight of the breasts as the area of the ligaments where they are suspended. It is known as the strain $(\sigma)$, which is the force exerted divided to the cross sectional area of the application. So, if we have an apple suspended of a stem, the gravitational force exerted down on the apple will be its weight $\left(\mathrm{F}_{\mathrm{g}}=\mathrm{mg}\right)$ and the cross sectional area would be the area of the circle of the stem shown in the above figure. The area of the circle is equal to $\mathrm{A}=\pi \mathrm{r}^{2}$, where $\mathrm{r}$ is the radius of the circle shown in the last figure. In the case of the breast, the stem would be analogous to the Cooper's ligaments and the breast to the apple. Therefore, the strain in this case is given by the next equation:

$\sigma=\frac{F_{g}}{A} \quad \mathbf{E q . 1}$

The reason that it depends on the strain is that any weight or gravitational force is distributed over the entire cross-sectional area that supports it. If the apple is suspended from the stem without falling (which means that the strain ( "no the weight") is not large enough to bring down the apple) and if the weight of the apple is duplicated and also the area of the stem, we have that the same strain is done on the apple and therefore it would not fall. The equation is as follows:

strain $=\frac{2 * \text { Weight }}{2 * \text { Area }} \quad \mathbf{E q . 2}$

So, the number 2 is simplified and we obtain:

strain $=\frac{\text { Weight }}{\text { Area }} \quad \mathbf{E q . 3}$

Even in ancient times, many "illusionists" or "wizards" used this principle to surprise people in quills bed. A person lay on a bed made of many quills putting different weights on his chest. The person does not experience anything because of the physics laws and so all the weight is distributed in each of the quills that form a large area in the bed. Obviously, if the person were sustained with a small number of quills, the area would be small and the strain would be large. So, the quills themselves would cause discomfort. This same set of relations of force and area occurs in the apple and in the breast. If the weight of the breasts is big and the area of the ligaments is small, then it would be a large strain and the breasts would fall. For other hand, if the yield strength of the ligaments is exceeded at some point, the breast would permanently fallen.

Besides, when the breasts bounce during a sport exercise, the ligaments can also be stretched or broken. It is because also the strain is proportional to the displacement of the breast (as we shall see later and as we said before the strain and no the weight, due to the fact that the bodies do not weigh when they fall). It can happen that during a moment the yield strength is exceeded (if there is a large vertical movement, for example when sport exercise is done), and so the breast could not regain its original position. However, a good bra breasts can minimize this effect (17).

The breasts are not immune to the laws of gravity and if there is not a proper support for them, ligaments and delicate tissues begin to stretch and distend. Already at an advanced age, most women's breasts are squashed and elongate, and for this reason it is necessary to use fasteners. 
The enlargement of the breasts is due to the accumulation of the fat around the mammary glands. The size is correlated with the amount of the fat tissue in the breast (Hytten and Leicht, 1971 and Niefert, 1985). In general, women who use a bra size type B have an average of five to seven pounds on her breasts, while women that use a size type $\mathrm{D}$ have $15-23$ pounds in their breasts.

One of the possible reasons for the shrinkage and sagging breasts is the involution. Breast involution is a process where the milk production system ends. Then, the breasts shrink. It happens after pregnancy or during the process of accustoming to drink milk or during menopause. When the tissue inside the breast shrinks, and the skin around it does not, the breast can look empty and fallen. Then, fat is deposited in the woman's body (which takes months) and so, the breast can gain size, but in the most of the cases the fall remains.

Another reason for sagging breasts is when a woman loses weight. In this case, some fat disappears. The skin and ligaments of the breasts not retract simultaneously, and then the breast falls. It is possible to avoid with a good nutrition for the skin. There are some studies that show that the firmness of the breasts is closely related to sexual reproductive value of women ("nubility hypothesis"). The shape and size of breasts is an honest signal of residual reproductive value. The "nubility hypothesis" (14) proposes that the process of enlargement is due to sexual selection.

\section{Physiology of the Breasts}

The breast is an inhomogeneous structure containing many layers of many different types of tissue. The two predominant types of tissue within the breasts are fat and glandular tissue, which support breastfeeding. The surface layer (fascia) is separated from the skin by 0.5 to $2.5 \mathrm{~cm}$ of subcutaneous fat. Cooper's ligaments are extensions of fibrous tissue as extended tentacles in all directions of the fascia (top layer) to the skin $(1,2,4,8)$.

The firmness of the breasts depends of the Cooper ligament. In adult mammary glands, they are 15 to 20 irregular lobes converging to the nipples through pipelines from 2 to $4.5 \mathrm{~mm}$ in diameter (4). These ducts are surrounded by dense connective tissue. These fibers act as a support $(4,6)$. It should be noted that breast carcinomas are accompanied by local changes in the mechanical properties of the breasts due to the change of stiffness as size and shape.

The structure of the breast is important because breast mechanism is affected by the structure and mechanical properties of its constituents. These change with the age, the physiological and pathological conditions, and disorders (1). An important anatomical review was given by Cooper (1840), while a microscopic description was given by Vorherr (1974).

\section{Theory}

\subsection{Modulus of Young}

If a sample of material of cross section $\mathrm{A}$, and initial length $\mathrm{y}_{\mathrm{o}}$ is subjected to a force $\mathrm{F}$ acting along its axis, it will suffer a stretch of magnitude $\Delta y$. It will depend on the force applied, the area $\mathrm{A}$ and the initial length $\mathrm{y}_{\mathrm{o}}(18,19)$ : 

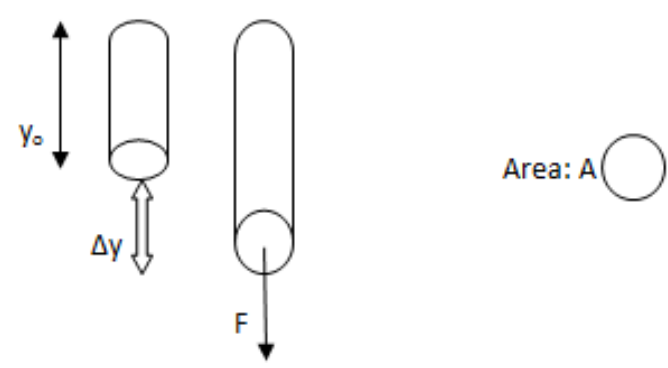

Fig.2 Stretching of a rod depending of $\Delta y, F, A$ and $y_{o}$

We analyze three cases for the rod and in all cases of the same material:

a) For the same area $\mathrm{A}$ and initial length $\mathrm{y}_{\mathrm{o}}$ and if the force $\mathrm{F}$ is increased, then $\Delta \mathrm{y}$ increases obviously.

b) Now if $y_{o}$ is kept constant and the area $\mathrm{A}$ is increased then a greater force is required if we want to produce the same displacement $\Delta y$ of case a). So, if Cooper's ligaments have a larger diameter (larger area), the breasts will stretch less due to the gravitational force.

c) Finally if the area A remains constant, but $y_{0}$ is smaller, then more force is required if we want to produce the same displacement of the case a) and b). Thus, if the breasts are smaller, it will be more difficult to stretch due to the gravitational force.

If $\Delta y / y_{o}$ is much less than 1, it is found experimentally (with the rod above example) that for a wide range of applied forces, $\Delta \mathrm{y}$ is proportional to the applied $\mathrm{F}$, to its initial length strength $\mathrm{y}_{\mathrm{o}}$ and inversely proportional to the cross section area A:

$\Delta y \infty \frac{F y_{o}}{A} \quad \mathbf{E q . 4}$

Robert Hooke noticed this experimental relationship and it is valid for a lot of materials. It becomes an equality by introducing a constant called the Young's modulus:

$E \frac{\Delta y}{y_{o}}=\frac{F}{A} \quad \mathbf{E q . 5}$

$\mathrm{E}$ is a constant depending on the elasticity of each material.

$\sigma=\frac{F}{A} \quad$ (nominal strain) $\quad$ Eq.6

$€=\frac{\Delta y}{y_{o}} \quad$ (rate of nominal displacement $) \quad$ Eq.7

The formula (5) is valid for the linear zone or proportionality between $\sigma$ and $\epsilon$ as we will be explained later. In a linear model, $\mathrm{E}$ is constant and it does not change with the strain and with the rate of displacement.

The Young's modulus $\mathrm{E}\left(\mathrm{Pa}=\mathrm{N} / \mathrm{m}^{2}\right)$ is an elastic constant needed to characterize the elastic properties of a material.

$\sigma=E € \quad$ Eq.8 $\quad\left(\right.$ where $\sigma=\frac{F}{A}$ and $\left.€=\frac{\Delta y}{y_{o}}, \frac{F}{A}=E \frac{\Delta y}{y_{o}}\right)$ 


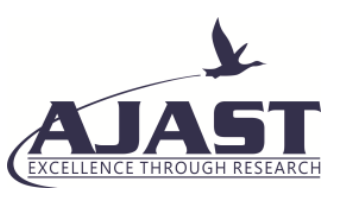

Asian Journal of Applied Science and Technology Volume 4, Issue 3, Pages 167-182, July-September 2020

Which is known as Hooke's law, valid only for the region of proportionality (linear region). In a linear model, the constant of the Young's modulus varies in an order of magnitude for several investigations. It is due to the methods of measurement or estimation. The lateral deformation rate to the axial is defined as the $v$ rate Poisson. For a very linear material, this rate ranges from 0.45 to 0.49 :

$v=\frac{\Delta x}{\Delta y} \quad$ Eq.9

If the model is not linear, then the formula to use is:

$E=\frac{\delta \sigma}{\delta \epsilon} \quad \mathbf{E q . 1 0}$

The symbol $\delta$ means partial derivative and its application is explained in an example later.

\subsection{Curve strain $(\sigma)$ versus deformation rate $(\epsilon)$}

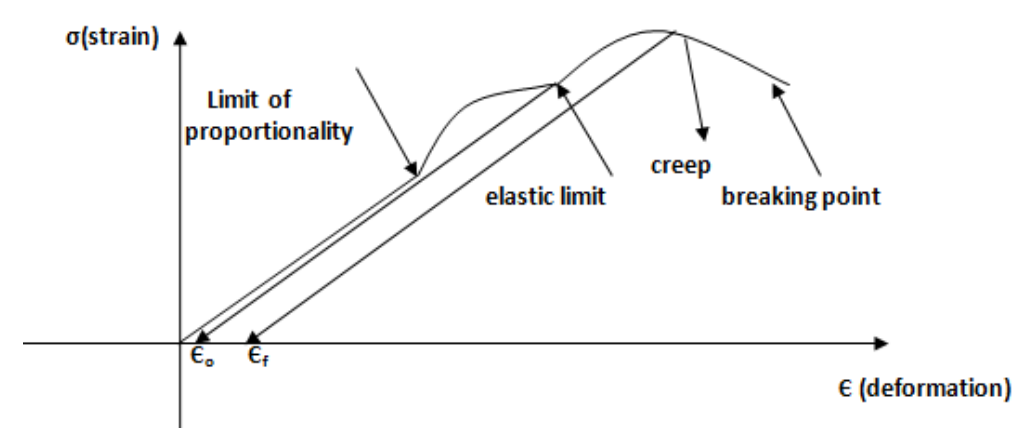

Fig.3 Relationship between the applied strain $\sigma$ and unit deformation $€$

As we can see in the figure, firstly we have the area where the proportionality equation $\sigma=\mathrm{E} €$ is fulfilled. The deformation is proportional to the strain, zone of validity of Hooke's Law. The slope of the straight line represents the value of Young's modulus E. Finally, the material reaches the limit value of proportionality. If the material is subjected to a strain until this value, after removing this strain, the material returns to its original shape without permanent deformation. Thus, the object will return to its original position if the applied force is removed.

If the material is subjected to a strain that exceeds the limit of proportionality, the graph deviates from the line of proportionality and no longer we have a linear relationship between $\sigma$ and $\epsilon$.

Nevertheless, if the strain does not exceed the elastic limit, the object will return to its original position. Thus, the applied strain does not cause permanent deformation. Usually, it is required that the elastic limit Co is less than 0.0001 .

If the object is subjected to an strain that exceeds the elastic limit of the material, then it enters to the plastic zone and in this zone the object will not return to its original position when the applied force is removed. Thus, it will be permanently deformed. If a strain is applied to the material that goes beyond the elastic limit, then it enters the creep area, where the material is easily deformed without increasing the strain. If we want that the material is not broken, it is necessary does not exceed the creep point. The creep point is defined as the intersection of the curve $\sigma$ versus $C$ and a line parallel to the linear zone passing the yield point (universally adopted) $\mathrm{Cf}=0.002(0.2 \%)$. 
Asian Journal of Applied Science and Technology Volume 4, Issue 3, Pages 167-182, July-September 2020

Generally, these values of $\sigma$ and $\epsilon$ (resulting from the intersection of the line) are chosen as the strain and the rate of deformation where it is considered that there is permanent deformation $\left(€ f=0,2 \%, €=\frac{\Delta y}{y_{o}}\right)(18)$.

\subsection{Relationship models for breast young $(2,7,9)$}

1) This model is the simplest. The tissue is modeled as linear and with Young's modulus of $1 \mathrm{kPa}$ for fat and glandular tissue. The linear relationship used is given by the formula 5) or 8) $\sigma=\mathrm{EC}$.

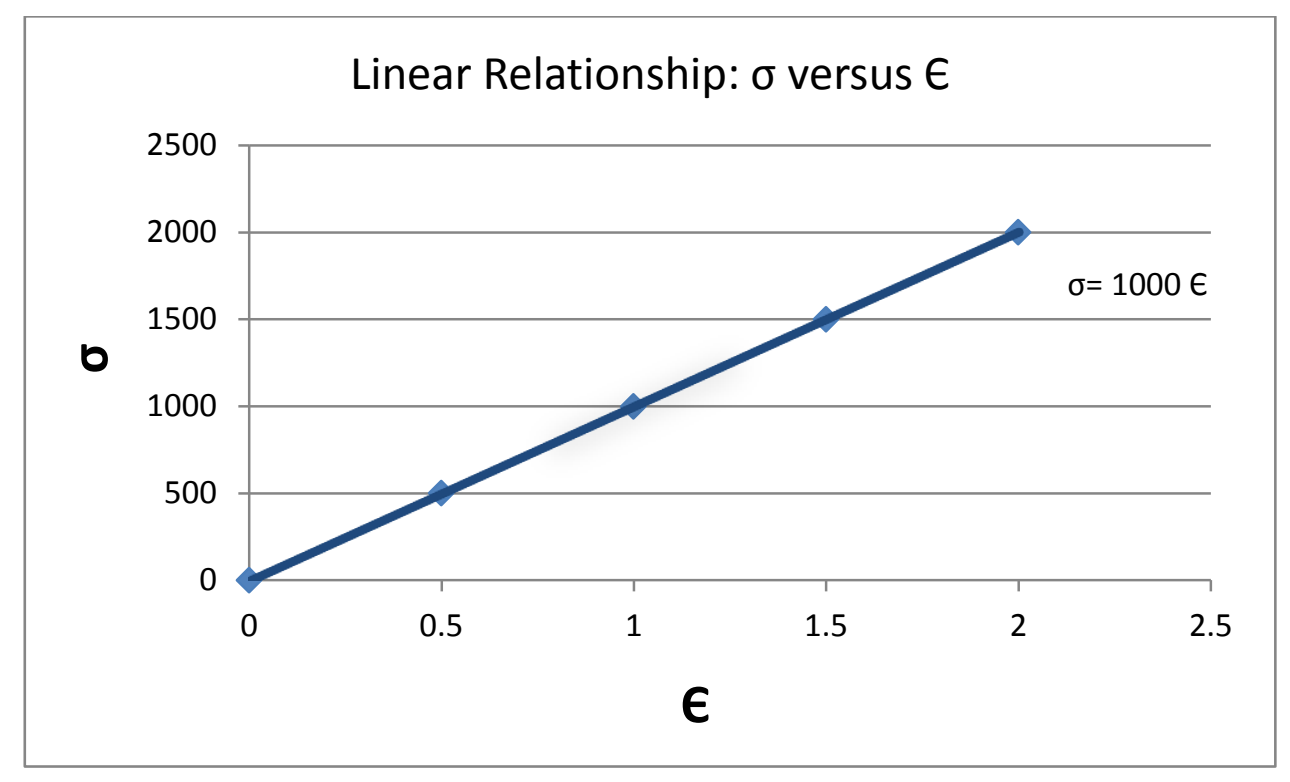

Fig.4 Linear relationship $\sigma$ versus $€$

2) Model used by Schnabel. It was modeled for fat and glandular tissue as in 1). But a modulus of $88 \mathrm{kPa}$ was chosen for the skin. The formula to use is also 5) or 8).

3) The linear model is the same as 2) but incorporating a Young's modulus of $10 \mathrm{kPa}$ for glandular tissue. The formula used is 5) or 8).

4) Nonlinear model proposed by Azar $(5,6,9)$. It is based on an exponential model taking into account the mechanical properties of the breast determined by Wellman. Then, it is done the change for the Young's modulus for the fat. It is taken into account the glandular tissue, the structures of the fibers, including Cooper's ligaments. The formula is:

$E=b e^{m \varepsilon} \quad \mathbf{E q . 1 1}$

Where, $b$ and $m$ are parameters determined for each tissue type given by Wellman: $b_{\text {gland }}=15.1 \mathrm{kPa}, \mathrm{m}_{\text {gland }}=10.0 \mathrm{Lb}$ $\mathrm{b}_{\mathrm{fat}}=4.46 \mathrm{kPa}, \quad \mathrm{m}_{\mathrm{fat}}=7.4 \mathrm{Lb}$

Data for the exponential graph:

$\mathrm{E}=\mathrm{be}^{\mathrm{m} \epsilon}$

$\mathrm{b}($ gland $)=15100 \mathrm{~Pa}$ 
$\mathrm{m}($ gland $)=4.5 \mathrm{~kg}$

$E=\partial \sigma / \partial \epsilon$

$\sigma=(\mathrm{b} / \mathrm{m}) *\left(\mathrm{e}^{\mathrm{mE}}-1\right)$

$b(f a t)=4460 \mathrm{~Pa}$

$\mathrm{m}(\mathrm{fat})=3.35 \mathrm{~kg}$
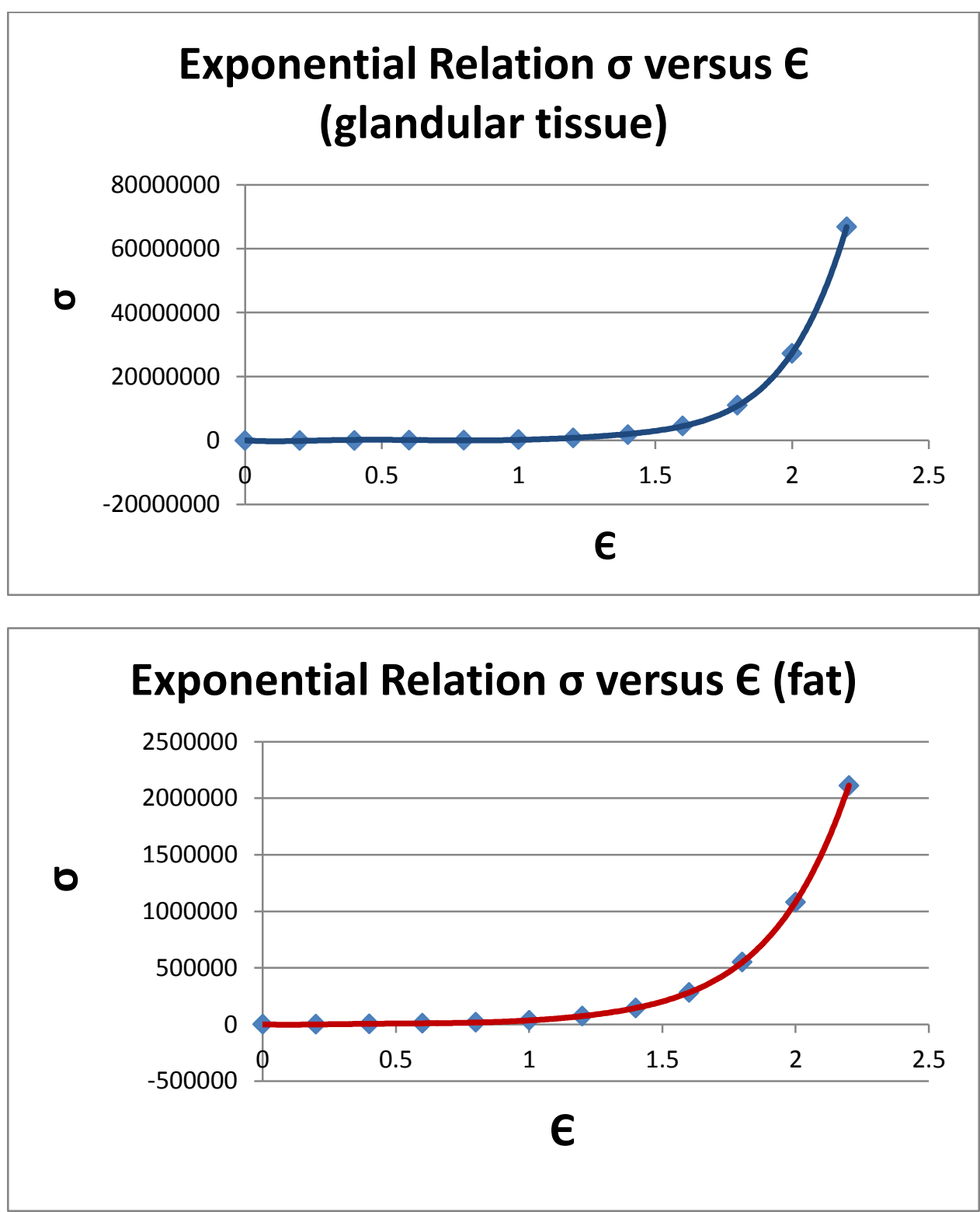

Fig.5 Exponential relationship $\sigma$ versus $C$ with the data for the gland, for the fat, the pattern of the graph is similar (blue: glandular tissue, red: fat)

With the modification of Azar:

$$
\begin{array}{lll}
E_{f a t}=\frac{E_{\text {glandavg }}-b_{\text {fat }}}{\epsilon_{\text {lim }}} \epsilon_{\text {fat }}+b_{\text {fat }} & \text { if } \epsilon_{\mathrm{fat}}<\epsilon_{\mathrm{lim}} & \text { Eq.12 } \\
E_{\text {fat }}=E_{\text {gland_avg }} & \text { if } \epsilon_{\mathrm{fat}} \geq \epsilon_{\mathrm{lim}} & \text { Eq.13 }
\end{array}
$$

Where, $E_{\text {gland_avg }}$ is an average Young's modulus for glandular tissue and for 
$\epsilon_{\text {limt }}=25 \% \quad E_{\text {gland_avg }}=184 \mathrm{kPa}$.

$E_{\text {fat }}=\frac{184000-4460}{0.25} \epsilon_{f a t}+4460$

$\sigma=359080 \epsilon^{2}+4460 €$

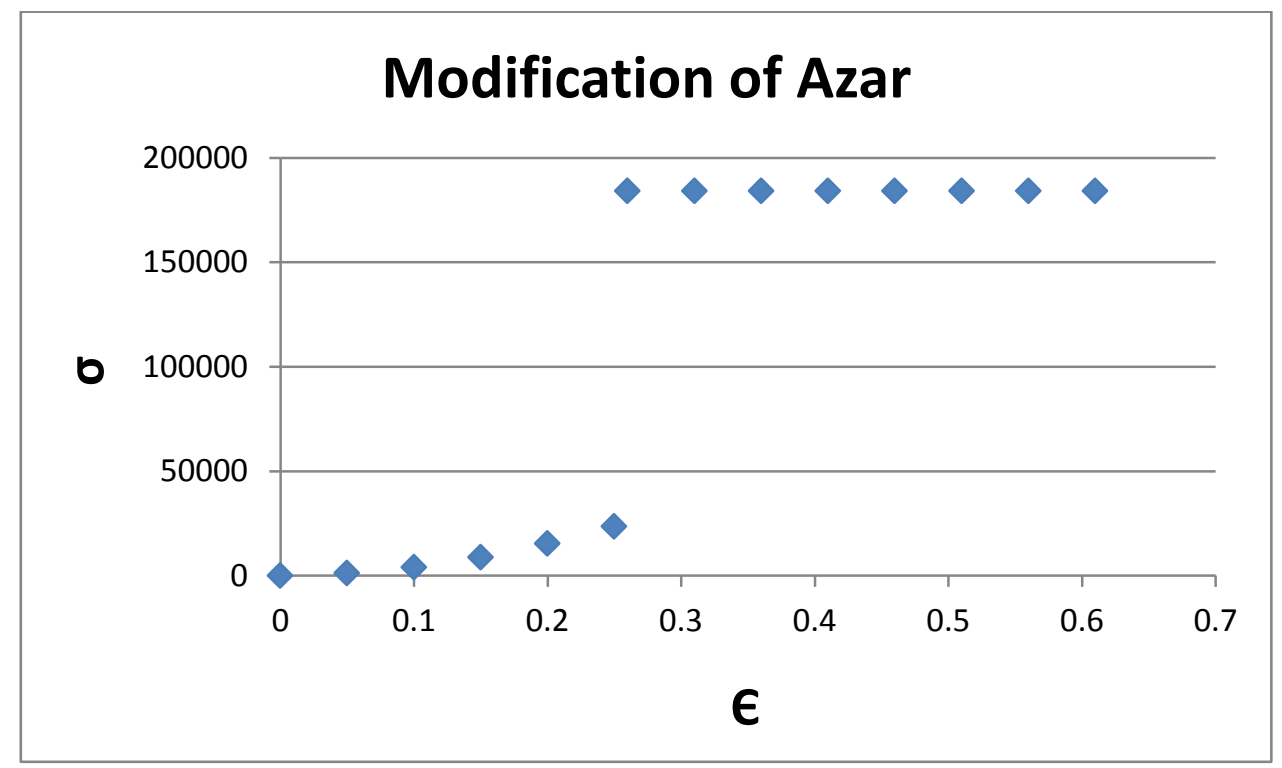

Fig.6 Graph $\sigma$ versus $C$ with the modification of Azar for the fat

5) The proposed nonlinear model by Samani (5) is also based on the mechanical properties of the breast determined by Wellman. However, the formula is obtained by fitting a polynomial equation of second and third order to the data of Wellman of the Young's modulus (E is in units of GPa).

$\mathrm{E}_{\text {fat }}=0.5197 \epsilon^{2}+0.0024 €+0.0049 \quad \mathbf{E q . 1 4}$

$E_{\text {gland }}=123.889 \epsilon^{3}-11.7667 \epsilon^{2}+0.6969 €+0.0121 \quad$ Eq.15

$\sigma_{\text {fat }}=0,173 \epsilon^{3}+0,0012 \epsilon^{2}+0,0049 \epsilon \quad$ Eq.16

$\sigma_{\text {gland }}=30,97 \epsilon^{4}-3,92 \epsilon^{3}+0,348 \epsilon^{2}+0,0121 \epsilon \quad$ Eq.17

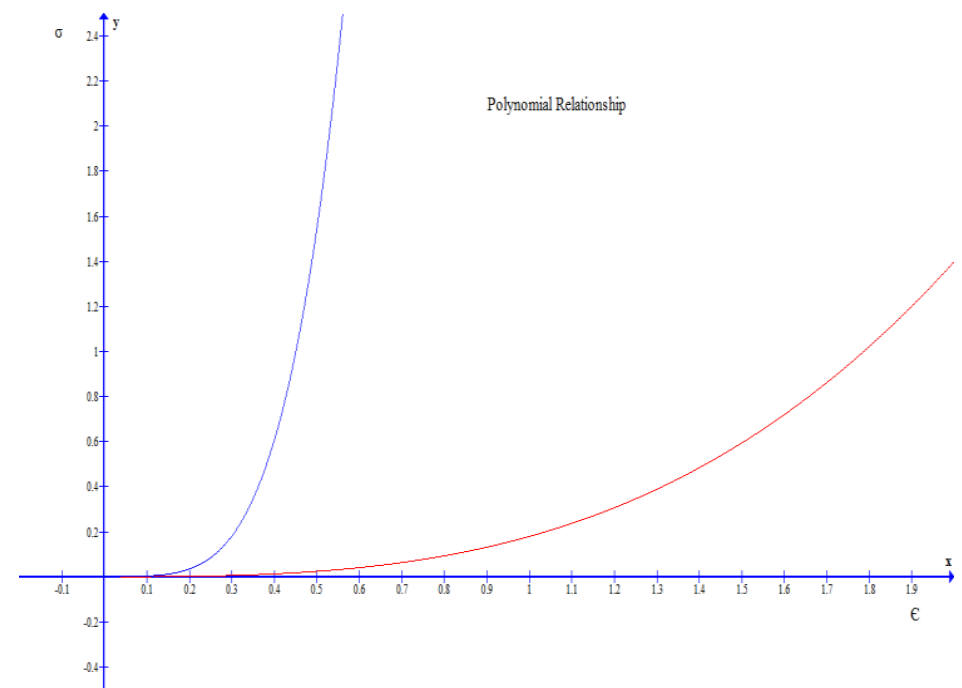

Fig.7 Relationship polynomial $\sigma$ versus $€$ for fat (red) and glandular tissue (blue) 


\subsection{Data table of Important Characteristics of the Breast}

\subsubsection{Table of thickness percentage of each constituent of the breast (8)}

\section{Tissue type}

Subcutaneous fat

Intraglandular fat

Retromammary fat

Total fat tissue

Glandular tissue / fibrous
\% thickness

8

Ductal system: $10-15 \%$ of the total mass

Adipose tissue: the remaining $85 \%$

Types of tissues of the breast: glandular, fibrous and adipose (fat)

3.4.2 Young's modulus (E) of the parts of the breast as an average of a number of samples (Samani, 2007)

The following table shows the Young's modulus (mechanical property) of 169 breast samples (Samani, 2007) (1). However, the Young's modulus is not sufficient to characterize the nonlinear behavior of the breasts, hence we must use also models, simulations and experimentation.

\begin{tabular}{|l|c|c|}
\hline Breast type & \# of samples & Young's modulus (kPa) \\
\hline Normal fat & & mean \pm STD \\
\hline Normal glandular tissue & 71 & $3.25 \pm 0.91$ \\
\hline Fibroadenoma & 26 & $3.24 \pm 0.61$ \\
\hline Infiltrating ductal carcinoma of low grade & 16 & $6.41 \pm 2.86$ \\
\hline Infiltrating lobular carcinoma & 4 & $10.40 \pm 2.60$ \\
\hline Infiltrating carcinoma "in situ" & 4 & $15.62 \pm 2.64$ \\
\hline Fibrocystic disorder & 4 & $16.38 \pm 1.55$ \\
\hline Infiltrating ductal carcinoma of intermediate & 21 & $42.11 \pm 7.35$ \\
grade & 9 & 20.21 \\
\hline Infiltrating ductal carcinoma high grade & 1 & 4.45 \\
\hline Invasive carcinoma & 169 & \\
\hline Necrosis & $9.52 \pm 12.47$ \\
\hline Total & & \\
\hline
\end{tabular}


Asian Journal of Applied Science and Technology Volume 4, Issue 3, Pages 167-182, July-September 2020

The stiffness values of the breast of the Table 3.4.2 ) cannot be generalized, because the morphology of the breast varies depending on the physiological conditions and the age of a woman. The morphology of the breast is highly variable among different women.

3.4.3 Table of Young's modulus of the parts of the breast given by Wellman, Krouskop and Samani (12)

\begin{tabular}{|c|c|c|c|c|c|}
\hline & & & & $\begin{array}{l}\text { E (kPa) Stiffness } \\
\text { glandular tissue }\end{array}$ & \\
\hline Author & Condition exp. & $\begin{array}{c}\text { Linear elastic model } \\
\text { (EL) }\end{array}$ & Fat & & Tumor \\
\hline Wellman & Identing & $\begin{array}{c}\text { EL and exponential } \\
\text { relation }\end{array}$ & $\begin{array}{c}17.4 \pm 8.4(15 \% \\
\text { def. })\end{array}$ & $\begin{array}{c}271.8 \pm 167.7(15 \% \\
\text { def. })\end{array}$ & $\begin{array}{c}2162 \text { ductal } \\
\text { carcin. }\end{array}$ \\
\hline Krouskop & Compression load & & $\begin{array}{c}20 \pm 8(20 \% \\
\text { comp. })\end{array}$ & $\begin{array}{c}48 \pm 15(20 \% \\
\text { comp. })\end{array}$ & $290 \pm 67$ \\
\hline Samani & Compression load & $\begin{array}{l}\text { EL and polynomial } \\
\text { function }\end{array}$ & $\begin{array}{c}3.25 \pm 0.9(5 \% \\
\text { comp.) }\end{array}$ & $\begin{array}{c}3.24 \pm 0.61(5 \% \\
\text { comp.) }\end{array}$ & $16.38 \pm 1.55$ \\
\hline
\end{tabular}

\subsubsection{Breast size table for different patients $(1,13)$}

$\begin{array}{lllllll}\text { Patient: } & 1 & 2 & 3 & 4 & 5 & 6\end{array}$

Breast size (mm): 210x90x130 225x117x135 178x117x156 250x108x98 205x126x84 148xN/Ax123

3.4.5 Normal tension and strain of three sensors located in different parts of the breast (11)

Sensor \#

1

2

3
Force (N)

$58.8 \times 10-3$

$9.81 \times 10-3$

$88.3 \times 10-3$

\section{Strain (N/m2)}

364.5

60.8

547.4

\section{Theoretical calculations}

The height of the breasts is about $12.67 \mathrm{~cm}$ (between 8 and $13 \mathrm{~cm}$ ) with a horizontal projection of the sines of 4.93 $\mathrm{cm}(4 \times 10 \mathrm{~cm})($ Westreich$)$.

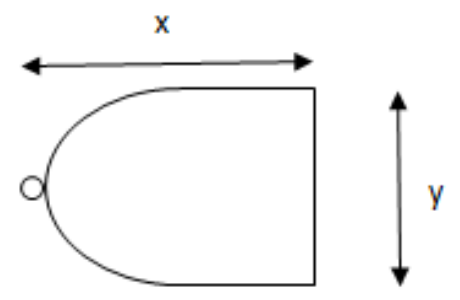

Fig.8 Height and horizontal projection of the breast 


\section{AJAST}

Asian Journal of Applied Science and Technology Volume 4, Issue 3, Pages 167-182, July-September 2020

An estimate of the breast deformation using linear theory is given by the formula (5), where by working out the rate $\mathrm{C}=\Delta \mathrm{y} / \mathrm{y}$, we obtain: $\frac{\Delta y}{y}=\frac{F}{A E}$

Where, $\varepsilon=\Delta y / y$ is the rate of the vertical deformation, $F$ is the gravitational force, $A$ is the cross sectional area of the breast (similar to the stem area holding the apple) and $\mathrm{E}$ is the Young's modulus $\left(\mathrm{N} / \mathrm{m}^{2}\right.$ ), which depends on the material and it is a measure of the level of elasticity of each material. In some cases, $\mathrm{E}$ is a constant and in others $\mathrm{E}$ varies with the rate of deformation $\varepsilon$.
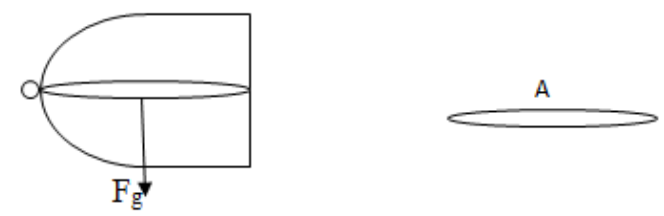

Fig.9 Gravitational force on the sinus and the transversal area of the sinus

We have the gravitational force $\mathrm{F}_{\mathrm{g}}$ :

$\mathrm{F}_{\mathrm{g}}=\mathrm{mg} \quad$ Eq.18

$F_{g}=F \quad$ Eq.19

Thus, the right side of the equation 19 is:

$\frac{F}{A E}=\frac{m g}{A E} \quad \mathbf{E q . 2 0}$

For other hand, the density of an object is given by the next equation:

$\rho=\frac{m}{V} \quad \mathbf{E q} .21$

Where, $\rho$ is the density of the object, $\mathrm{m}$ is the mass and $\mathrm{V}$ is the volume.

Nevertheless, the volume is given by the next equation (where we approximate the breast to a cylinder of high $y$ and area $\mathrm{A})$ :

V=Ay $\quad$ Eq.22

Therefore, by replacing the equation 22) in (21), we obtain:

$m=\rho A y \quad \mathbf{E q . 2 3}$

And by replacing $\mathrm{m}$ in the equation 20 ), we have:

$\frac{m g}{A E}=\frac{\rho A y g}{A E} \quad$ Eq.24

Finally, by replacing the equation 24) in (20) and it in the equation (5), we have:

$\frac{\Delta y}{y}=\frac{\rho A g y}{A E} \quad ; \frac{\Delta y}{y}=\frac{\rho g y}{E} \quad$ Eq.25

Where, $y$ is the vertical length of the breast. 
If we want to estimate $\Delta y$, the vertical displacement of the breast, we need to know the values of the breast density, the initial vertical length of the breast and an estimated value of the Young's modulus E of the breast.

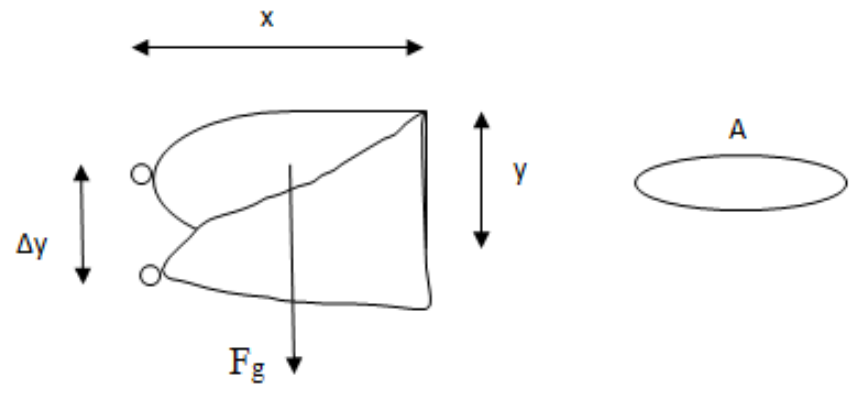

Fig.10 Vertical displacement of the breast due to the action of the gravitational force

We can use the typical value of the breast height $10 \mathrm{~cm}: 0.1 \mathrm{~m}$ and assuming $\rho=940 \mathrm{~kg} / \mathrm{m}^{3}$ (density of the water is about $1000 \mathrm{~kg} / \mathrm{m}^{3}$ and the material of the breast is very close to water), $\mathrm{g}=9.8 \mathrm{~m} / \mathrm{s}^{2}$ and $\mathrm{E}=3240 \mathrm{~N} / \mathrm{m} 2$ (the average Young's modulus of the breasts, table 3.4.2 and 3.4.3). By replacing these values in the equation 25), we obtain a displacement rate equal to the value $(C=\Delta y / y): C=0.3$.

By working out the variable $\Delta y$ from the equation $C=\Delta y / y$ and by replacing $y=10 \mathrm{~cm}$, we obtain:

\section{$\Delta \mathrm{y}=€ \mathrm{y} \quad \mathbf{E q . 2 6}$}

$\Delta y=3 \mathrm{~cm}$

The softness and firmness of the breasts are important mechanical properties that affect breast shape.

It is extremely important to analyze the equation (25), by working out $\Delta y$ :

$\Delta y=\frac{\rho g y^{2}}{E} \mathbf{E q} .27$

As we can see at the equation that $\Delta \mathrm{y}$ is directly proportional to $\mathrm{y}^{2}(7)$ and $\mathrm{g}, \rho$ and $\mathrm{E}$ are constants in our analysis (if we analyze the same woman but with different vertical heights of the breast, then the density and Young's modulus would be the same). Although E may vary considerably from one woman to another, firstly we analyze it constant in the same woman with different heights of the breast.

Thus, if the value of $y$ is small (small breasts) the deformation would be less and by doing a calculation, we could verify it. For example, for $y=8 \mathrm{~cm}$ and by replacing in the equation 27) the same values of $\rho(940 \mathrm{~kg} / \mathrm{m} 3)$ and $\mathrm{E}$ (3240 N / m2), we obtain: $\Delta y=1,8 \mathrm{~cm}$

Now, the breast density does not vary much from one woman to another. If the parameter E varies considerably and affects the shape of the breasts, then high values of $\mathrm{E}$ (high rigidity, firm breasts), give small values of $\Delta y$ and small values of $\mathrm{E}$ (very soft breasts) give large values of $\Delta \mathrm{y}$. The deformation of the breast increases for very soft breasts and decrements for very firm breasts. Thus, a woman can have large breasts (y large) but if the breasts are very firm (E large), then there is not significant deformation of the breast. If the breasts are soft and large, then there is a considerable deformation of the breast because $\mathrm{y}$ is large (large breasts) and $\mathrm{E}$ is small (soft breasts). It is possible to observe at the equation 27). 
Therefore, if the woman has small breasts, then she does not have to worry much if her breasts are soft or firm, because as $\Delta y$ depends on $\mathrm{y}^{2}$ (this relationship is stronger than the ratio $1 / \mathrm{E}$ of the equation). If the breasts are small, $\mathrm{y}$ is small and as $\Delta \mathrm{y}$ is proportional to $\mathrm{y}^{2}$, then $\Delta \mathrm{y}$ is very small.

For example, for $\mathrm{y}=5 \mathrm{~cm}$, with $\rho=940 \mathrm{~kg} / \mathrm{m}^{3}$ and $\mathrm{E}=3240 \mathrm{~N} / \mathrm{m}^{2}$ and by using the equation 27 ), we obtain: $\Delta \mathrm{y}=0,7$ $\mathrm{cm}$

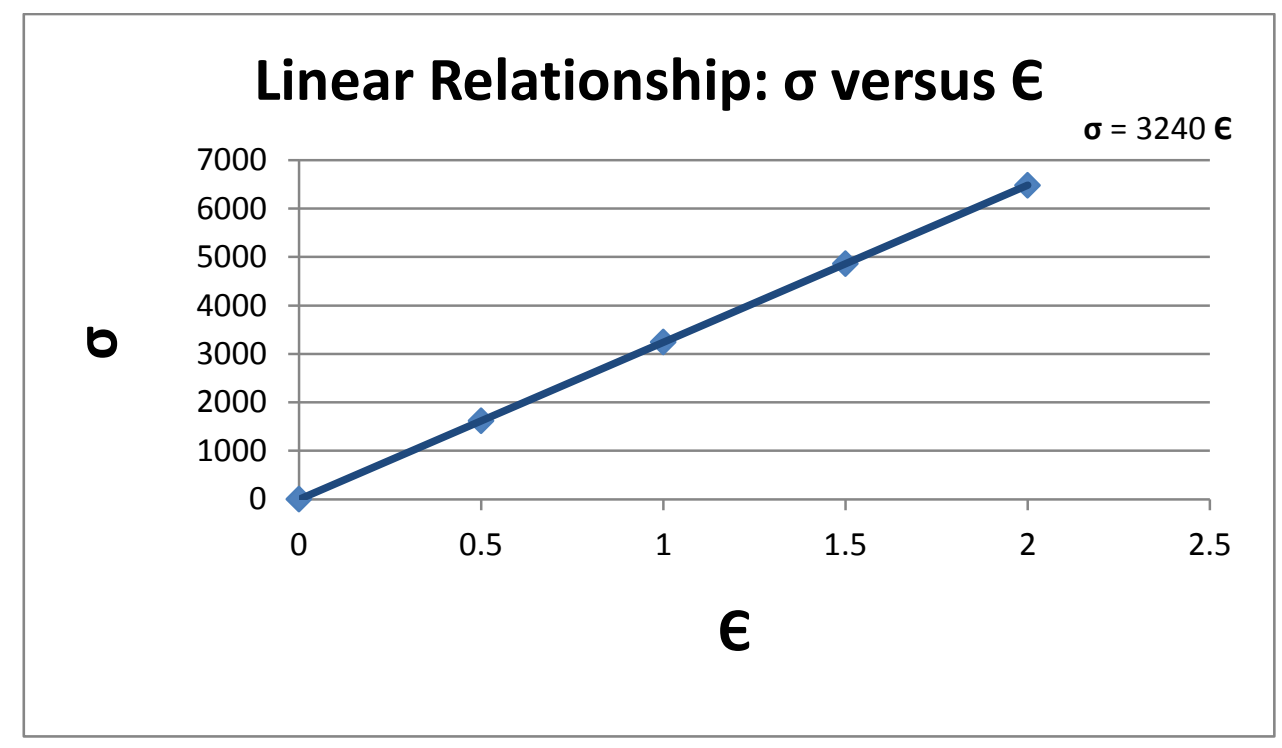

Fig.11 Linear relationship $\sigma$ versus $C$ for $E=3240 \mathrm{~N} / \mathrm{m}^{2}$ (glandular tissue)

\section{Simulation and Experimentation (phantom) of the biomechanics of breasts}

Biomechanical models developed using the Finite Element Method (FEM) and programming can be used to model the relationship between different types of tissues and with the application of forces or movements ( 1 .. $.9,11,12,13,15,16)$. It can help predict mechanical deformations of the tissue during medical operations and derive and quantify the properties of the tissue with the observed deformations (15). The theory of finite elasticity (10) is used to implement the finite element model.

For other hand, as we said before, it is possible to use the finite element models (FEM) to perform simulations of the deformation of the breast due to different test weights. We need to know the biomechanical properties, boundary conditions and a configuration of a normal breast and deformed. It can be very useful when it is done a diagnostic examination (mammography, magnetic resonance) and the patient moves from one position to another and the breasts are compressed. Thus, masses of difficult observation could be visible between those images. It may be possible to have a biomechanical model of the breast.

Then, it can be used for breast cancer diagnosis and for studies of the gravity effects on light and heavy breasts and to study the effects of compression on radiographic tests.

Furthermore, it is possible to use silicone gel phantom (1), (3), for breast experimentation. So, the breast could be subjected to different weights and analyze the elasticity of the breast to support different forces. It can be done together with the respective simulations. Therefore, we can compare the experimental results (vertical 
displacements) with the simulations. Thus, one could validate the biomechanical model and the simulation program. In addition to the phantom and simulations, we could make a graph of $\sigma$ versus $C$ like the figure 3 . Finally, we can get the limit points of yield. Besides with these researches, we can design fasterners (17) or other material with the aim that the breast does not reach the yield point.

\subsection{History of experimental models and application of the finite element models to the breast (1)}

Rivlin (1950) developed an analytical solution for the general case of identifying the deformed state of a cylinder with thick walls made of the Mooney-Rivlin material under the action of pressure and torque.

Gould developed a model for the heart (1972). For the breast, models have been developed only in the last 20 years.

Neifert Yu (1995) was the first to model the movement of the breasts to determine the applicability of holographic interferometry in the detection of breast cancer. Holographic interferometry is a technique that creates an interference pattern that is produced when a reference image and a distorted image which are remembered in the same holographic film. He created a 3D image using the finite element model to predict deformations due to different weights.

Kita (1998) created a model of deformation due to compression in mammographic tests.

Azar (2000) developed a model by using the finite element theory to observe deformations during MRI (magnetic resonance) tests of the breast. The procedure contrasts images uncompressed and compressed and then use the finite element theory to predict a tumor in the compressed area.

Wellman (1999) conducted experiments in vivo from a variety of tissue samples (fat, glandular and different types of carcinomas) and it is a good source to cite when the mechanical properties of the breasts are required

Samani (2001) used the finite deformation theory to model breast compression.

Tanner (2001) presents a method with quantitative information improving the accuracy of biomechanical models in the deformation prediction due to the compression.

Schnabel (2001) validated the technique used by Tanner by recording images generated from finite element models simulating tissue displacements. Also, Ruiter (2002) developed a linear elastic model to achieve the precise location of tumors.

\section{Conclusions}

The level of the breast deformation is not related to the fact that the breasts are large or small as it was mentioned before.

For large breasts, it depends if the breasts are very soft (small E, high $\Delta y$ ) or firm (high E, small $\Delta y$ ) (Young's modulus E) in order that a significant deformation occurs. We apply the equation: $\Delta y=\frac{\rho g y^{2}}{E}$ in order to see the relation between $\Delta \mathrm{y}$ and $\mathrm{E}$.

For small breasts, there is generally no significant deformation. 
Asian Journal of Applied Science and Technology Volume 4, Issue 3, Pages 167-182, July-September 2020

The deformation of the breast increases for very soft breasts and decrements for very firm breasts.

A good bra of breast can minimize this effect.

\section{Bibliography}

1) Modelling Breast Tissue Mechanics under Gravity Loading, Vijayaraghavan Rajagopal, Bioengineering Institute, Universtiy of Auckland, New Zealand, January 2007.

2) The Comparison of Biomechanical Breast Models: Initial Results, C. Tanner, J. A. Schnabel, A. Castellano Smith, L.I. Sonoda, D.L.G. Hill, D.J. Hawkes, Division of Radiological Sciences and Medical Engineering, King's College London, UK.

3) Biomechanical Models of the Breast Tracking Breast Cancer Across Medical Images, V. Rajagopal, Auckland Bioengineering Institute, 2008.

4) Methods for Modeling and Predicting Mechanical Deformations of the Breast during Interventional Procedures, Fred S. Azar, Dimitris N. Metaxas, Reid T. Miller, Mitchell D. Schnall, Dept. of BioEngineering, University of Pennsylvania, 1999

5) A Deformable Finite Element Model of the Breast for Predicting Mechanical Deformations under External Perturbations, Fred S. Azar, Dimitris N. Metaxas, Mitchell D. Schnall, 2001.

6) A Finite Element Model of the Breast for Predicting Mechanical Deformations during Biopsy Procedures, Fred S. Azar, Dimitris N. Metaxas, Mitchell D. Schnall, 2000.

7) Non-linear modeling of breast tissue, Jonathan P. Whiteley and David J. Gavaghan, Oxford University Computing Laboratory, University of Oxford, 2007.

8) Three Dimensional, in silico breast phantom for multimodality image simulations, Michael Mahr, University of Illinois, 2009.

9) Methods for modeling and predicting mechanical deformations of the breast under external perturbations, Fred S. Azar, Dimitris N. Metaxas and Mitchell D. Schnall, University of Pennsylvania, 2001.

10) The Static Theory of Finite Elasticity, A. J. M. Spencer, Department of Theoretical Mechanics, University of Nottingham, 1969.

11) Biomechanics of Breast Tissue: Preliminary study of force deformation relationship, Celeste Williams, Bradley Clymer, Petra Schmalbrock, Biomedical Engineering Center, University of Ohio.

12) Modeling breast biomechanics for multi-modal image analysis-successes and challenges, V. Rajagopal, Poul M. F. Nielsen, Martyn P. Nash, 2009.

13) Finite Element Modelling of Breast Biomechanis: Predicting the effects of gravity, Vijay Rajagopal, Jae-Hoon Chung, Ruth Warren, Ralph Highnam, Martyn Nash, Poul Nielsen, Computational Biomechanics for Medicine, Copenhagen, Denmark, 2006. 
14) The Nubillity Hypothesis, the human breast as an honest signal of residual reproductive value, Frank Marlowe, University of California, 1997.

15) Predicting Tumour Location by Modelling the Deformation of the Breast, P. Pathmanathan, J. Whiteley, S. J. Chapman, D. Gavaghan, and J. M. Brady, University of Oxford, 2008.

16) Biomechanics of Breast Tissue: Preliminary study of shape deformation of normal breast tissue, C. Williams, B. Clymer, P. Schmalbrock, K. Fujimura, Biomedical Engineering Center, University of Ohio.

17) Biomechanical Analysis of a Prototype Sports Bra, Cathy Starr, University of Oklahoma, 2005.

18) Introducción a la teoría de la Elasticidad, S. Gil, UNSAM.

19) Física de Cutnell, Segunda Edición, John Wiley \& Song, Inc., 2004. 